\title{
Oxidation Reactions of the Posphinidene Oxide Ligand
}

\author{
María Alonso, M. Angeles Alvarez, M. Esther García, Miguel A. Ruiz*, Hayrullo Hamidov, ${ }^{\dagger}$ and John C. \\ Jeffery. $^{\dagger}$ \\ Departamento de Química Orgánica e Inorgánica/IUQOEM, Universidad de Oviedo, 33071 Oviedo, Spain. ${ }^{\dagger}$ School of \\ Chemistry, University of Bristol, Bristol BS8 ITS, UK.
}

\section{Supporting Information}

\author{
Preparative Procedures and Microanalytical Data for New Compounds. $\left(R^{*}=2,4,6-C_{6} \mathbf{H}_{2}{ }^{t} \mathrm{Bu}_{3}\right)$
}

\section{General Procedures and Starting Materials}

All manipulations and reactions were carried out under a nitrogen (99.995\%) atmosphere using standard Schlenk techniques. Solvents were purified according to literature procedures, ${ }^{1}$ and distilled prior to use. Petroleum ether refers to that fraction distilling in the range $65-70 \quad{ }^{\circ} \mathrm{C}$. Compound $(\mathrm{H}-$ $\mathrm{DBU})\left[\mathrm{MoCp}(\mathrm{CO})_{2}\left\{\mathrm{P}(\mathrm{O}) \mathrm{R}^{*}\right\}\right](\mathbf{1}),{ }^{2}\left(\mathrm{R}^{*}=2,4,6-\mathrm{C}_{6} \mathrm{H}_{2}{ }^{\mathrm{t}} \mathrm{Bu}_{3} ; \mathrm{Cp}=\eta^{5}-\right.$ $\mathrm{C}_{5} \mathrm{H}_{5}, \mathrm{DBU}=1,8$-diazabicyclo [5.4.0] undec-7-ene), $\left[\mathrm{FeCp}_{2}\right] \mathrm{BF}_{4},{ }^{3}$ and dimethyldioxirane ${ }^{4}$ were prepared as described previously. All other reagents were obtained from the usual commercial suppliers and used as received. Chromatographic separations were carried out using jacketed columns cooled by tap water (ca. $285 \mathrm{~K}$ ), or by $2-$ propanol in a closed circuit, kept at the required temperature with a cryostat. Commercial aluminium oxide (activity I, 150 mesh) was degassed under vacuum prior to use. The latter was mixed under nitrogen with the appropriate amount of water to reach the activity desired. Filtrations were performed using diatomaceous earth unless otherwise stated. IR stretching frequencies of CO ligands are given in $\mathrm{cm}^{-1}$, were measured in solution and are referred to as $v_{\mathrm{CO}}$ (solvent). Nuclear Magnetic Resonance (NMR) spectra were routinely recorded at $300.13\left({ }^{1} \mathrm{H}\right), 121.50\left({ }^{31} \mathrm{P}\left\{{ }^{1} \mathrm{H}\right\}\right)$ or $75.47 \mathrm{MHz}$ $\left({ }^{13} \mathrm{C}\left\{{ }^{1} \mathrm{H}\right\}\right)$ at $290 \mathrm{~K}$ in $\mathrm{CD}_{2} \mathrm{Cl}_{2}$ solutions unless otherwise stated. Chemical shifts $(\delta)$ are given in ppm, relative to internal tetramethylsilane (TMS) or external $85 \%$ aqueous $\mathrm{H}_{3} \mathrm{PO}_{4}$ solutions $\left({ }^{31} \mathrm{P}\right)$. Coupling constants $(J)$ are given in Hertz. Assignments of the ${ }^{13} \mathrm{C}$ NMR resonances for the aryl group follow the labelling shown in the figure below, and are reported as $\mathrm{C}^{\mathrm{n}}\left(\mathrm{C}_{6} \mathrm{H}_{2}\right)$ or $\mathrm{C}^{\mathrm{m}}\left({ }^{\mathrm{t}} \mathrm{Bu}\right)$ as required $(\mathrm{n}=1$ to $6 ; \mathrm{m}=1,2)$.

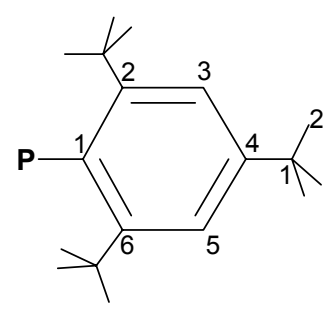

Preparative procedures, spectroscopic and microanalytical data for the new compounds.

Preparation of $\left[\mathrm{Mo}_{2} \mathrm{Cp}_{2}(\mathbf{C O})_{4}\left\{\mathbf{P}(\mathrm{O}) \mathbf{R}^{*}\right\}_{2}\right] \quad$ (2). Solid $\left[\mathrm{FeCp}_{2}\right] \mathrm{BF}_{4}(0.008 \mathrm{~g}, 0.030 \mathrm{mmol})$ was added to a dichloromethane solution $(10 \mathrm{~mL})$ of compound $1(0.020 \mathrm{~g}, 0.030 \mathrm{mmol})$ at $238 \mathrm{~K}$, and the mixture was stirred for $1 \mathrm{~min}$ to give a red solution. The solvent was then removed in vacuum, the residue extracted with toluene / petroleum ether (2/1) and the extracts filtered. The solvents were then removed under vacuum and the residue crystallized by slow diffusion of a layer of petroleum ether into a concentrated dichloromethane solution of the product to give compound 2 as red crystals $(0.027 \mathrm{~g}, 93 \%)$. Anal. Calcd for $\mathrm{C}_{50} \mathrm{H}_{68} \mathrm{Mo}_{2} \mathrm{O}_{6} \mathrm{P}_{2}: \mathrm{C}, 58.94 ; \mathrm{H}, 6.73$. Found: $\mathrm{C}, 59.40 ; \mathrm{H}, 6.93 . v_{\mathrm{CO}}$ $\left(\mathrm{CH}_{2} \mathrm{Cl}_{2}\right): 1918(\mathrm{~m}, \mathrm{sh}), 1901(\mathrm{~s}) .{ }^{1} \mathrm{H}$ NMR: $\delta 7.46\left(\mathrm{~d}, J_{\mathrm{HP}}=3,2 \mathrm{H}\right.$, $\left.\mathrm{C}_{6} \mathrm{H}_{2}\right), 5.01(\mathrm{~s}, 5 \mathrm{H}, \mathrm{Cp}), 1.62\left(\mathrm{~s}, 18 \mathrm{H}, \mathrm{CH}_{3}\right), 1.34\left(\mathrm{~s}, 9 \mathrm{H}, \mathrm{CH}_{3}\right)$. ${ }^{31} \mathrm{P}\left\{{ }^{1} \mathrm{H}\right\}$ NMR: $\delta 463.5$ (s).

Spectroscopic data for $\left[\mathbf{W}_{\mathbf{2}} \mathbf{C p}_{2}(\mathbf{C O})_{4}\{\mathbf{P}(\mathbf{O}) \mathbf{R}\}_{2}\right] . \quad v_{\mathrm{CO}}$ $\left(\mathrm{CH}_{2} \mathrm{Cl}_{2}\right): 1912(\mathrm{~m}), 1892$ (s). ${ }^{1} \mathrm{H}$ NMR: $\delta 7.48$ (s, $\left.2 \mathrm{H}, \mathrm{C}_{6} \mathrm{H}_{2}\right), 5.10$ (s, $5 \mathrm{H}, \mathrm{Cp}), 1.61\left(\mathrm{~s}, 18 \mathrm{H}, \mathrm{CH}_{3}\right), 1.34\left(\mathrm{~s}, 9 \mathrm{H}, \mathrm{CH}_{3}\right) .{ }^{31} \mathrm{P}\left\{{ }^{1} \mathrm{H}\right\}$ NMR: $\delta$ $404.5\left(\mathrm{~s}, J_{\mathrm{PW}}=380\right)$.

Preparation of $\left[\mathrm{MoCp}\left\{\boldsymbol{\kappa}^{2}-\mathrm{OP}\left(\mathrm{OC}_{6} \mathrm{H}_{4} \mathrm{OH}\right) \mathrm{R}^{*}\right\}(\mathrm{CO})_{2}\right] \quad$ (3). Solid $p$-benzoquinone $(0.005 \mathrm{~g}, 0.046 \mathrm{mmol})$ was added to a tetrahydrofuran solution $(10 \mathrm{~mL})$ of compound $\mathbf{1}$ at $223 \mathrm{~K}$, and the mixture was stirred for $1 \mathrm{~h}$ while allowing it to reach room temperature slowly. Solvent was then removed under vacuum from the violet resulting solution, and the residue was chromatographed on alumina (activity IV) at $285 \mathrm{~K}$. Elution with tetrahydrofuran /petroleum ether (1/4) gave a pink fraction which yielded, after removal of solvents in vacuum, compound $\mathbf{3}$ as a pink microcrystalline solid $(0.029 \mathrm{~g}, 90 \%)$. The crystals used in the Xray study were grown by slow diffusion of petroleum ether into a dichloromethane solution of the complex at $253 \mathrm{~K}$. Anal. Calcd for $\mathrm{C}_{31} \mathrm{H}_{39} \mathrm{MoO}_{5} \mathrm{P}: \mathrm{C}, 60.19 ; \mathrm{H}, 6.36$. Found: $\mathrm{C}, 60.69 ; \mathrm{H}, 6.57$. $v_{\mathrm{CO}}$ $\left(\mathrm{CH}_{2} \mathrm{Cl}_{2}\right): 1954$ (s), 1865 (s). ${ }^{1} \mathrm{H}$ NMR $(200.13 \mathrm{MHz}): \delta 7.52$ (dd, $\left.J_{\mathrm{HP}}=6, J_{\mathrm{HH}}=2,1 \mathrm{H}, \mathrm{C}_{6} \mathrm{H}_{2}\right), 7.42\left(\mathrm{t}, J_{\mathrm{HP}}=J_{\mathrm{HH}}=2,1 \mathrm{H}, \mathrm{C}_{6} \mathrm{H}_{2}\right), 7.01$ $\left(\mathrm{AA}^{\prime} \mathrm{XX}^{\prime} \mathrm{Z}, J_{\mathrm{HH}}+J_{\mathrm{H}^{\prime} \mathrm{H}}=9, J_{\mathrm{PH}}=2,2 \mathrm{H}, \mathrm{C}_{6} \mathrm{H}_{4}\right), 6.79\left(\mathrm{AA}^{\prime} \mathrm{XX}^{\prime}, J_{\mathrm{HH}}\right.$ $\left.+J_{\mathrm{H}^{\prime} \mathrm{H}}=9,2 \mathrm{H}, \mathrm{C}_{6} \mathrm{H}_{4}\right), 5.14(\mathrm{~s}, 1 \mathrm{H}, \mathrm{OH}), 5.01(\mathrm{~s}, 5 \mathrm{H}, \mathrm{Cp}), 1.75,1.57$, $1.35\left(3 \times \mathrm{s}, 3 \times 9 \mathrm{H}, \mathrm{CH}_{3}\right) \cdot{ }^{31} \mathrm{P}\left\{{ }^{1} \mathrm{H}\right\}$ NMR: $\delta 74.5 .{ }^{13} \mathrm{C}\left\{{ }^{1} \mathrm{H}\right\}$ NMR: $\delta$ $252.7\left(\mathrm{~d}, J_{\mathrm{CP}}=30, \mathrm{CO}\right), 245.6(\mathrm{~s}, \mathrm{CO}), 156.8\left[\mathrm{~d}, J_{\mathrm{CP}}=26, \mathrm{C}^{2,6}(\mathrm{Ar})\right]$, $154.3\left[\mathrm{~s}, \mathrm{C}^{4}(\mathrm{Ar})\right], 153.0\left[\mathrm{~s}, \mathrm{C}^{4}\left(\mathrm{C}_{6} \mathrm{H}_{4}\right)\right], 152.6\left[\mathrm{~d}, J_{\mathrm{CP}}=3, \mathrm{C}^{6,2}(\mathrm{Ar})\right]$, $145.5\left[\mathrm{~d}, J_{\mathrm{CP}}=8, \mathrm{C}^{1}\left(\mathrm{C}_{6} \mathrm{H}_{4}\right)\right], 130.7\left[\mathrm{~d}, J_{\mathrm{CP}}=53, \mathrm{C}^{1}(\mathrm{Ar})\right], 124.0[\mathrm{~d}$, $\left.J_{\mathrm{CP}}=13, \mathrm{C}^{3,5}(\mathrm{Ar})\right], 123.8\left[\mathrm{~d}, J_{\mathrm{CP}}=17, \mathrm{C}^{5,3}(\mathrm{Ar})\right], 122.9\left[\mathrm{~d}, J_{\mathrm{CP}}=3\right.$, $\left.\mathrm{C}^{2}\left(\mathrm{C}_{6} \mathrm{H}_{4}\right)\right], 116.2\left[\mathrm{~s}, \mathrm{C}^{3}\left(\mathrm{C}_{6} \mathrm{H}_{4}\right)\right], 95.5(\mathrm{~s}, \mathrm{Cp}), 40.1,35.2,33.8$ [3 x s, $\left.3 \times \mathrm{C}^{1}\left({ }^{4} \mathrm{Bu}\right)\right], 34.2,32.9,30.9\left[3 \times \mathrm{x}, 3 \times \mathrm{C}^{2}\left({ }^{\mathrm{t}} \mathrm{Bu}\right)\right]$.

Preparation of cis$\left[\operatorname{MoBrCp}(\mathrm{CO})_{2}\left\{\mathbf{P}(\mathrm{OH})\left(\mathrm{CH}_{2} \mathrm{CMe}_{2} \mathrm{C}_{6} \mathrm{H}_{2}{ }^{t} \mathrm{Bu}_{2}\right\}\right]\right.$ (4). Bromine (16 $\mu \mathrm{L}$ of a $2.17 \mathrm{M}$ solution in dichloromethane, $0.035 \mathrm{mmol}$ ) was added to a dichloromethane solution $(10 \mathrm{~mL})$ of compound $\mathbf{1}(0.020$ $\mathrm{g}, 0.030 \mathrm{mmol}$ ) at $203 \mathrm{~K}$ to give instantaneously an orange solution. The solvent was then removed in vacuum, the residue extracted with toluene / petroleum ether (1/1) and the extracts filtered using a 
cannula. The solvents were then removed in vacuum and the residue crystallized by slow diffusion of a layer of petroleum ether into a concentrated dichloromethane solution of the product to give compound 4 as red, X-ray quality crystals (0.026 g, $78 \%$ ). Anal. Calcd for $\mathrm{C}_{25} \mathrm{H}_{34} \mathrm{BrMoO}_{3} \mathrm{P}$ : C, 50.95; H, 5.81. Found: C, 51.41; H, 5.95. $v_{\mathrm{CO}}\left(\mathrm{CH}_{2} \mathrm{Cl}_{2}\right): 1972$ (vs), 1894 (s). ${ }^{1} \mathrm{H}$ NMR (200.13 MHz): $\delta$ $7.56\left(\mathrm{dd}, J_{\mathrm{HP}}=5, J_{\mathrm{HH}}=2,1 \mathrm{H}, \mathrm{C}_{6} \mathrm{H}_{2}\right), 7.22\left(\mathrm{t}, J_{\mathrm{HP}}=J_{\mathrm{HH}}=2,1 \mathrm{H}\right.$, $\left.\mathrm{C}_{6} \mathrm{H}_{2}\right), 6.86(\mathrm{~s}, 1 \mathrm{H}, \mathrm{OH}), 5.50(\mathrm{~s}, 5 \mathrm{H}, \mathrm{Cp}), 2.66\left(\mathrm{ABX}, J_{\mathrm{HH}}=14, J_{\mathrm{HP}}\right.$ $\left.=13,1 \mathrm{H}, \mathrm{CH}_{2}\right), 2.34\left(\mathrm{ABX}, J_{\mathrm{HH}}=14, J_{\mathrm{HP}}=11,1 \mathrm{H}, \mathrm{CH}_{2}\right), 1.46$, $1.39\left(2 \times \mathrm{s}, 2 \times 3 \mathrm{H}, \mathrm{CMe}_{2}\right), 1.48,1.35\left(2 \times \mathrm{s}, 2 \times 9 \mathrm{H}, \mathrm{CH}_{3}\right) .{ }^{31} \mathrm{P}\left\{{ }^{1} \mathrm{H}\right\}$ NMR: $\delta 132.7$.

Preparation of (H-DBU)[MoCp(CO) $\left.\left\{\boldsymbol{\kappa}^{2}-\mathrm{OP}(\mathrm{O}) \mathrm{R}^{*}\right\}\right](5)$. Dimethyldioxirane $(1.0 \mathrm{~mL}$ of a ca. $0.1 \mathrm{M}$ solution in acetone, 0.10 $\mathrm{mmol})$ was added to a dichloromethane solution $(10 \mathrm{~mL})$ of compound $1(0.030 \mathrm{~g}, 0.045 \mathrm{mmol})$ at $243 \mathrm{~K}$, and the mixture was stirred for $20 \mathrm{~min}$ to give an orange solution. The solvent was then removed in vacuum, the residue extracted with diethyl ether (15 $\mathrm{mL}$ ) and the extract filtered using a cannula. Removal of the solvent in vacuum and washing of the residue with petroleum ether gave compound $\mathbf{5}$ as an air-sensitive, reasonably pure orange powder $(0.016 \mathrm{~g}, 52 \%)$. Attempts to further purify this product resulted in its progressive decomposition. $v_{\mathrm{CO}}\left(\mathrm{CH}_{2} \mathrm{Cl}_{2}\right): 1904$ (vs), 1803 (s). ${ }^{1} \mathrm{H}$ NMR (200.13 MHz): $\delta 11.78$ (s, 1H, NH), 7.34 (dd, $\left.J_{\mathrm{HP}}=5, J_{\mathrm{HH}}=2,1 \mathrm{H}, \mathrm{C}_{6} \mathrm{H}_{2}\right), 7.24\left(\mathrm{t}, J_{\mathrm{HP}}=J_{\mathrm{HH}}=2,1 \mathrm{H}, \mathrm{C}_{6} \mathrm{H}_{2}\right), 4.81$ (s, 5H, Cp), 3.47-3.20 (m, 6H, $\left.\mathrm{CH}_{2}\right), 2.79,1.96(2 \times \mathrm{m}, 2 \times 2 \mathrm{H}$, $\mathrm{CH}_{2}$ ), 1.76, 1.52, 1.32 (3 x s, 3 x 9H, $\left.\mathrm{CH}_{3}\right), 1.75-1.64\left(\mathrm{~m}, 6 \mathrm{H}, \mathrm{CH}_{2}\right)$. ${ }^{31} \mathrm{P}\left\{{ }^{1} \mathrm{H}\right\}$ NMR: $\delta 39.6$. Figures $\mathrm{S} 1$ and $\mathrm{S} 2$ show typical IR and ${ }^{31} \mathrm{P}$ NMR spectra of the complex. The phosphorus resonance appearing at $15.3 \mathrm{ppm}$ is due to partial decomposition of $\mathbf{5}$ always occurring upon manipulation of its solutions.

Preparation of (H-DBU)[MoCp(CO) $\left.2\left\{\kappa^{2}-\mathrm{SP}(\mathrm{O}) \mathrm{R}^{*}\right\}\right](6)$. Sulphur $(0.73 \mathrm{~mL}$ of a ca. $0.062 \mathrm{~N}$ solution in dichloromethane, $0.045 \mathrm{mmol})$ was added to a dichloromethane solution $(10 \mathrm{~mL})$ of compound $1(0.030 \mathrm{~g}, 0.045 \mathrm{mmol})$ at $273 \mathrm{~K}$, and the mixture was stirred for $2 \mathrm{~min}$ to give an orange solution. The solvent was then removed in vacuum, the residue extracted with diethyl ether $(2 \times 10$ $\mathrm{mL}$ ) and the extracts filtered. Removal of the solvent in vacuum and washing of the residue with petroleum ether gave compound 6 as an orange powder $(0.029 \mathrm{~g}, 93 \%)$. Anal. Calcd for $\mathrm{C}_{34} \mathrm{H}_{51} \mathrm{MoN}_{2} \mathrm{O}_{3}$ PS: C, 58.78; H, 7.40; N, 4.03. Found: C, 59.34; H, 7.17; N, 4.12. $v_{\mathrm{CO}}\left(\mathrm{CH}_{2} \mathrm{Cl}_{2}\right): 1911$ (vs), 1816 (s). ${ }^{1} \mathrm{H}$ NMR: $\delta 12.28$ $(\mathrm{s}, 1 \mathrm{H}, \mathrm{NH}), 7.21\left(\mathrm{dd}, J_{\mathrm{HP}}=5, J_{\mathrm{HH}}=2,1 \mathrm{H}, \mathrm{C}_{6} \mathrm{H}_{2}\right), 7.09\left(\mathrm{t}, J_{\mathrm{HP}}=J_{\mathrm{HH}}\right.$ $\left.=2,1 \mathrm{H}, \mathrm{C}_{6} \mathrm{H}_{2}\right), 4.71(\mathrm{~s}, 5 \mathrm{H}, \mathrm{Cp}), 3.38-3.28\left(\mathrm{~m}, 6 \mathrm{H}, \mathrm{CH}_{2}\right), 2.75$, $1.94\left(2 \times \mathrm{m}, 2 \times 2 \mathrm{H}, \mathrm{CH}_{2}\right), 1.71,1.64,1.28$ ( $\left.3 \times \mathrm{s}, 3 \times 9 \mathrm{H}, \mathrm{CH}_{3}\right)$, 1.76-1.61 (m, 6H, $\left.\mathrm{CH}_{2}\right) .{ }^{31} \mathrm{P}\left\{{ }^{1} \mathrm{H}\right\}$ NMR: $\delta$ 83.8. ${ }^{13} \mathrm{C}\left\{{ }^{1} \mathrm{H}\right\}$ NMR: $\delta$ $254.6\left(\mathrm{~d}, J_{\mathrm{CP}}=29, \mathrm{CO}\right), 247.1(\mathrm{~s}, \mathrm{CO}), 165.7(\mathrm{~s}, \mathrm{CN}), 156.4\left[\mathrm{~d}, J_{\mathrm{CP}}\right.$ $\left.=21, \mathrm{C}^{2,6}(\mathrm{Ar})\right], 153.7\left[\mathrm{~s}, \mathrm{C}^{4}(\mathrm{Ar})\right], 148.4\left[\mathrm{~s}, \mathrm{C}^{6,2}(\mathrm{Ar})\right], 144.1\left[\mathrm{~d}, J_{\mathrm{CP}}=\right.$ $\left.46, \mathrm{C}^{1}(\mathrm{Ar})\right], 121.7\left[\mathrm{~d}, J_{\mathrm{CP}}=10, \mathrm{C}^{3,5}(\mathrm{Ar})\right], 120.4\left[\mathrm{~d}, J_{\mathrm{CP}}=15\right.$, $\mathrm{C}^{5,3}$ (Ar)], 94.2 (s, Cp), 53.2, 47.9, 38.2, 32.0, 29.0, 26.7, 24.4, 19.4 $\left(8 \times \mathrm{s}, \mathrm{CH}_{2}\right), 41.5,40.1,34.7\left[3 \times \mathrm{x}, 3 \times \mathrm{C}^{1}\left({ }^{\mathrm{t}} \mathrm{Bu}\right)\right], 34.8,34.3,31.2[3$ $\left.\mathrm{x} \mathrm{s}, 3 \times \mathrm{C}^{2}(\mathrm{Bu})\right]$.

Preparation of $\left[\mathrm{MoCp}\left\{\boldsymbol{\kappa}^{2}-(\mathrm{MeS}) \mathbf{P}(\mathrm{O}) \mathbf{R}^{*}\right\}(\mathrm{CO})_{2}\right]$ (7). Methyl iodide $(27 \mu \mathrm{L}, 0.43 \mathrm{mmol})$ was added to a dichloromethane solution $(10 \mathrm{~mL})$ of compound $6(0.030 \mathrm{~g}, 0.043 \mathrm{mmol})$ at $290 \mathrm{~K}$, and the mixture was stirred for $15 \mathrm{~min}$ to give a yellow solution. Solvent was then removed in vacuum and the residue was chromatographed on alumina (activity IV) at $253 \mathrm{~K}$. Elution with tetrahydrofuran /petroleum ether (1/3) gave a yellow fraction which yielded, after removal of solvents in vacuum, compound 7 as a yellow microcrystalline solid $(0.022 \mathrm{~g}, 92 \%)$. The crystals used in the X-ray study were grown from a concentrated petroleum ether solution of the complex at $253 \mathrm{~K}$. Anal. Calcd for $\mathrm{C}_{26} \mathrm{H}_{37} \mathrm{MoPO}_{3} \mathrm{~S}$ :
C, 56.12; H, 6.70. Found: C, 55.65; H, 6.56. $v_{\mathrm{CO}}\left(\mathrm{CH}_{2} \mathrm{Cl}_{2}\right): 1963$ (vs), 1887 (s). ${ }^{1} \mathrm{H} \mathrm{NMR}: \delta 7.33\left(\mathrm{dd}, J_{\mathrm{HP}}=6, J_{\mathrm{HH}}=2,1 \mathrm{H}, \mathrm{C}_{6} \mathrm{H}_{2}\right)$, $7.20\left(\mathrm{t}, J_{\mathrm{HP}}=J_{\mathrm{HH}}=2,1 \mathrm{H}, \mathrm{C}_{6} \mathrm{H}_{2}\right), 4.59(\mathrm{~s}, 5 \mathrm{H}, \mathrm{Cp}), 2.09\left(\mathrm{~d}, J_{\mathrm{HP}}=3\right.$, $\left.3 \mathrm{H}, \mathrm{SCH}_{3}\right), 1.67,1.46,1.30\left(3 \times \mathrm{s}, 3 \times 9 \mathrm{H}, \mathrm{CH}_{3}\right) .{ }^{31} \mathrm{P}\left\{{ }^{1} \mathrm{H}\right\} \mathrm{NMR}: \delta$ 102.0. ${ }^{13} \mathrm{C}\left\{{ }^{1} \mathrm{H}\right\}$ NMR: $\delta 242.1$ (d, $\left.J_{\mathrm{CP}}=40, \mathrm{CO}\right), 239.7$ (s, CO), $157.0\left[\mathrm{~d}, J_{\mathrm{CP}}=26, \mathrm{C}^{2,6}(\mathrm{Ar})\right], 152.3\left[\mathrm{~s}, \mathrm{C}^{4}(\mathrm{Ar})\right], 151.0\left[\mathrm{~s}, \mathrm{C}^{6,2}(\mathrm{Ar})\right]$, $143.0\left[\mathrm{~d}, J_{\mathrm{CP}}=28, \mathrm{C}^{1}(\mathrm{Ar})\right], 122.5\left[\mathrm{~d}, J_{\mathrm{CP}}=8, \mathrm{C}^{3,5}(\mathrm{Ar})\right], 122.3[\mathrm{~d}$, $\left.J_{\mathrm{CP}}=15, \mathrm{C}^{5,3}(\mathrm{Ar})\right], 93.7$ (s, Cp), 41.6, 39.9, 35.0 [3 x s, 3 x $\left.\mathrm{C}^{1}\left({ }^{\mathrm{t}} \mathrm{Bu}\right)\right], 34.2,33.4,30.9$ [3 x s, $\left.3 \times \mathrm{C}^{2}\left({ }^{\mathrm{t}} \mathrm{Bu}\right)\right], 19.0\left(\mathrm{~s}, \mathrm{SCH}_{3}\right)$.

Preparation of $\left[\mathrm{MoCp}\left\{\kappa^{2}-\mathrm{SP}(\mathrm{OMe}) \mathrm{R}^{*}\right\}(\mathrm{CO})_{2}\right]$ (8). Solid $\left(\mathrm{Me}_{3} \mathrm{O}\right) \mathrm{BF}_{4}(0.010 \mathrm{~g}, 0.067 \mathrm{mmol})$ was added to a dichloromethane solution $(10 \mathrm{~mL})$ of compound $6(0.040 \mathrm{~g}, 0.058 \mathrm{mmol})$ at $273 \mathrm{~K}$, and the mixture was stirred for $2 \mathrm{~min}$ to give an orange-yellow solution. Solvent was then removed in vacuum and the residue was chromatographed on alumina (activity IV) at $253 \mathrm{~K}$. Elution with dichloromethane /petroleum ether (1/9) gave a yellow fraction which yielded, after removal of solvents in vacuum, compound $\mathbf{8}$ as an orange microcrystalline solid $(0.016 \mathrm{~g}, 50 \%)$. Elution with tetrahydrofuran /petroleum ether (1/3) gave a yellow fraction which analogously yielded compound 7 as a yellow microcrystalline solid $(0.014 \mathrm{~g}, 44 \%)$. The crystals used in the X-ray study of compound 8 were grown by slow diffusion of petroleum ether into a dichloromethane solution of the complex at $253 \mathrm{~K}$. Anal. Calcd for $\mathrm{C}_{26} \mathrm{H}_{37} \mathrm{MoPO}_{3} \mathrm{~S}$ : C, 56.12; H, 6.70. Found: C, 56.58; H, 6.55. $v_{\mathrm{CO}}$ $\left(\mathrm{CH}_{2} \mathrm{Cl}_{2}\right): 1947$ (vs), 1862 (s). ${ }^{1} \mathrm{H} \mathrm{NMR}: \delta 7.37\left(\mathrm{dd}, J_{\mathrm{HP}}=6, J_{\mathrm{HH}}=\right.$ 2, $\left.1 \mathrm{H}, \mathrm{C}_{6} \mathrm{H}_{2}\right), 7.26\left(\mathrm{t}, J_{\mathrm{HP}}=J_{\mathrm{HH}}=2,1 \mathrm{H}, \mathrm{C}_{6} \mathrm{H}_{2}\right), 4.91(\mathrm{~s}, 5 \mathrm{H}, \mathrm{Cp})$, $3.18\left(\mathrm{~d}, J_{\mathrm{HP}}=16,3 \mathrm{H}, \mathrm{OCH}_{3}\right), 1.62,1.57,1.30\left(3 \mathrm{x} \mathrm{s}, 3 \times 9 \mathrm{H}, \mathrm{CH}_{3}\right)$. ${ }^{31} \mathrm{P}\left\{{ }^{1} \mathrm{H}\right\}$ NMR: $\delta$ 122.0. ${ }^{13} \mathrm{C}\left\{{ }^{1} \mathrm{H}\right\}$ NMR: $\delta 251.1\left(\mathrm{~d}, J_{\mathrm{CP}}=27, \mathrm{CO}\right)$, 239.4 (s, CO), $156.4\left[\mathrm{~d}, J_{\mathrm{CP}}=24, \mathrm{C}^{2,6}(\mathrm{Ar})\right], 156.7$ [s, $\left.\mathrm{C}^{4}(\mathrm{Ar})\right], 152.1$ $\left[\mathrm{d}, J_{\mathrm{CP}}=4, \mathrm{C}^{6,2}(\mathrm{Ar})\right], 131.8\left[\mathrm{~d}, J_{\mathrm{CP}}=66, \mathrm{C}^{1}(\mathrm{Ar})\right], 123.3\left[\mathrm{~d}, J_{\mathrm{CP}}=11\right.$, $\left.\mathrm{C}^{3,5}(\mathrm{Ar})\right], 122.5\left[\mathrm{~d}, J_{\mathrm{CP}}=18, \mathrm{C}^{5,3}(\mathrm{Ar})\right], 94.4(\mathrm{~s}, \mathrm{Cp}), 52.7\left[\mathrm{~d}, J_{\mathrm{CP}}=\right.$ 7, $\left.\mathrm{OCH}_{3}\right], 41.1,40.3\left[2 \times \mathrm{d}, J_{\mathrm{CP}}=4,2 \times \mathrm{C}^{1}\left({ }^{\mathrm{t}} \mathrm{Bu}\right)\right], 34.9\left[\mathrm{~s}, \mathrm{C}^{1}\left({ }^{\mathrm{t}} \mathrm{Bu}\right)\right]$, $33.8,35.3,30.9\left[3 \times\right.$ s, $\left.3 \times \mathrm{C} \mathrm{C}^{2}\left({ }^{\mathrm{t}} \mathrm{Bu}\right)\right]$.

\section{Crystallographic Studies}

X-ray structure determination for compounds 4 and 7 . Crystallographic data for compounds $\mathbf{4}$ and $\mathbf{7}$ are presented respectively in the CIF file format. In each case a single crystal was coated in high-vacuum grease and mounted on a glass fibre. X-ray measurements were made using a Bruker SMART CCD areadetector diffractometer with $\mathrm{Mo}-\mathrm{K}_{\alpha}$ radiation $(\lambda=0.71073 \AA){ }^{5}$ Intensities were integrated ${ }^{5}$ from several series of exposures, each exposure covering $0.3^{\circ}$ in $\omega$, and the total data set being a sphere (4) or hemisphere (7). Absorption corrections were applied, based on multiple and symmetry-equivalent measurements using SADABS. ${ }^{6}$ The structures were solved by direct methods (7) or Patterson synthesis (4), and all were refined by least squares on weighted $\mathrm{F}^{2}$ values for all reflections. ${ }^{7}$ All non-hydrogen atoms were assigned anisotropic displacement parameters and refined without positional constraints except the $C(52), C(53)$ and $C(54)$ atoms of the disordered ${ }^{\mathrm{t}} \mathrm{Bu}$ group in 4 . All hydrogen atoms were constrained to ideal geometries and refined with fixed isotropic displacement parameters. Hydrogen atom $\mathrm{H}(3)$ in compound 4 was located in the electron density difference map, assigned an isotropic displacement parameters and refined without positional constraints. Complex neutral-atom scattering factors were used. ${ }^{8}$

X-ray Structure Determination of Compounds 3 and 8 . Crystallographic data for compounds $3 \cdot \mathrm{CH}_{2} \mathrm{Cl}_{2}$ and $\mathbf{8}$ are presented respectively in the CIF file format. The $\mathrm{X}$-ray intensity data were collected on a Smart-CCD-1000 Bruker diffractometer using graphite-monochromated $\mathrm{Mo}_{\alpha} \mathrm{K}_{\alpha}$ radiation at $293 \mathrm{~K}$. Cell dimensions and orientation matrixes were initially determined from least-squares refinements on reflections measured in three sets of 
30 exposures collected in three different $\omega$ regions and eventually refined against all reflections. The software SMART ${ }^{5}$ was used for collecting frames of data, indexing reflections, and determining lattice parameters. The collected frames were then processed for integration by the software SAINT, ${ }^{5}$ and a multi-scan absorption correction was applied with SADABS. ${ }^{6}$ The structure was solved by Patterson interpretation and phase expansion, and was refined by full-matrix least squares on $F^{2}$ using SHELXL97. ${ }^{9}$ All nonhydrogen atoms were refined anisotropically. For compound $\mathbf{3}$ all hydrogen atoms were located in the Fourier map in the last leastsquares refinements and were refined with an overall isotropic thermal parameter except the hydroxyl atom $\mathrm{H}(1)$, engaged in hydrogen bonding with $\mathrm{O}(3)$, which was fixed at a calculated geometric position. For compound $\mathbf{8}$, all hydrogen atoms were fixed at calculated geometric positions and were given an overall isotropic thermal parameter.

\section{References}

(1) D. D. Perrin, W. L. F. Armarego, Purification of Laboratory Chemicals. (Pergamon Press: Oxford, U.K., 1998).

(2) Alonso, M; García, M. E.; Ruiz, M. A.; Hamidov, H.; Jeffery, J. C. J. Am. Chem. Soc. 2004, 126, 13610.

(3) Connelly, N. G.; Geiger, W. E. Chem. Rev. 1996, 96, 877.

(4) Adam, W.; Bialas, J.; Hadjiarapoglou, L. Chem. Ber. 1991, 124, 2377.

(5) SMART \& SAINT Software Reference Manuals, Version 5.051 (Windows NT Version); Bruker Analytical X-ray Instruments: Madison WI, 1998.

(6) G. M. Sheldrick. SADABS: A program for absorption correction with the Siemens SMART system; University of Gottingen: Germany, 1996.

(7) SHELXTL program system version 5.1; Bruker Analytical Xray Instruments Inc., Madison, WI, 1998.

(8) International Tables for Crystallography, Kluwer, Dordrecht, 1992, vol. C.

(9) Sheldrick, G. M. SHELXL97: Program for the Refinement of Crystal Structures; University of Göttingen, Göttingen, Germany, 1997. 


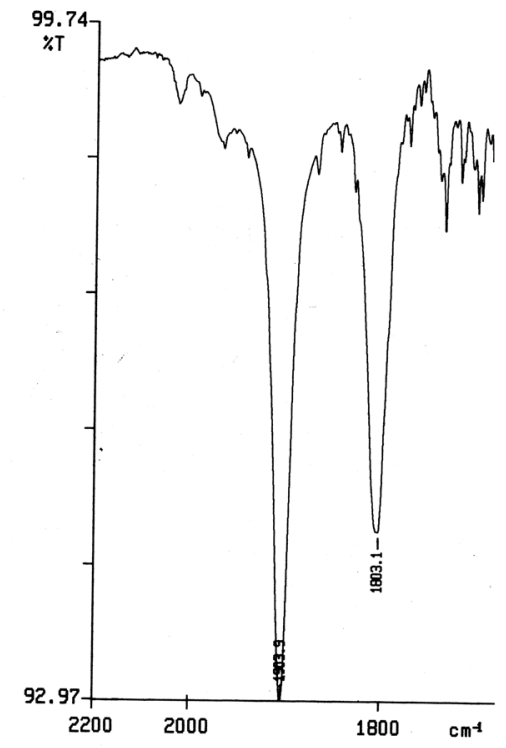

Figure S1. IR spectra (carbonyl region) of compound 5, recorded in dichloromethane solution.

$\boldsymbol{F}_{31 \mathrm{P} / \mathrm{CD} 2 \mathrm{Cl} 2}$

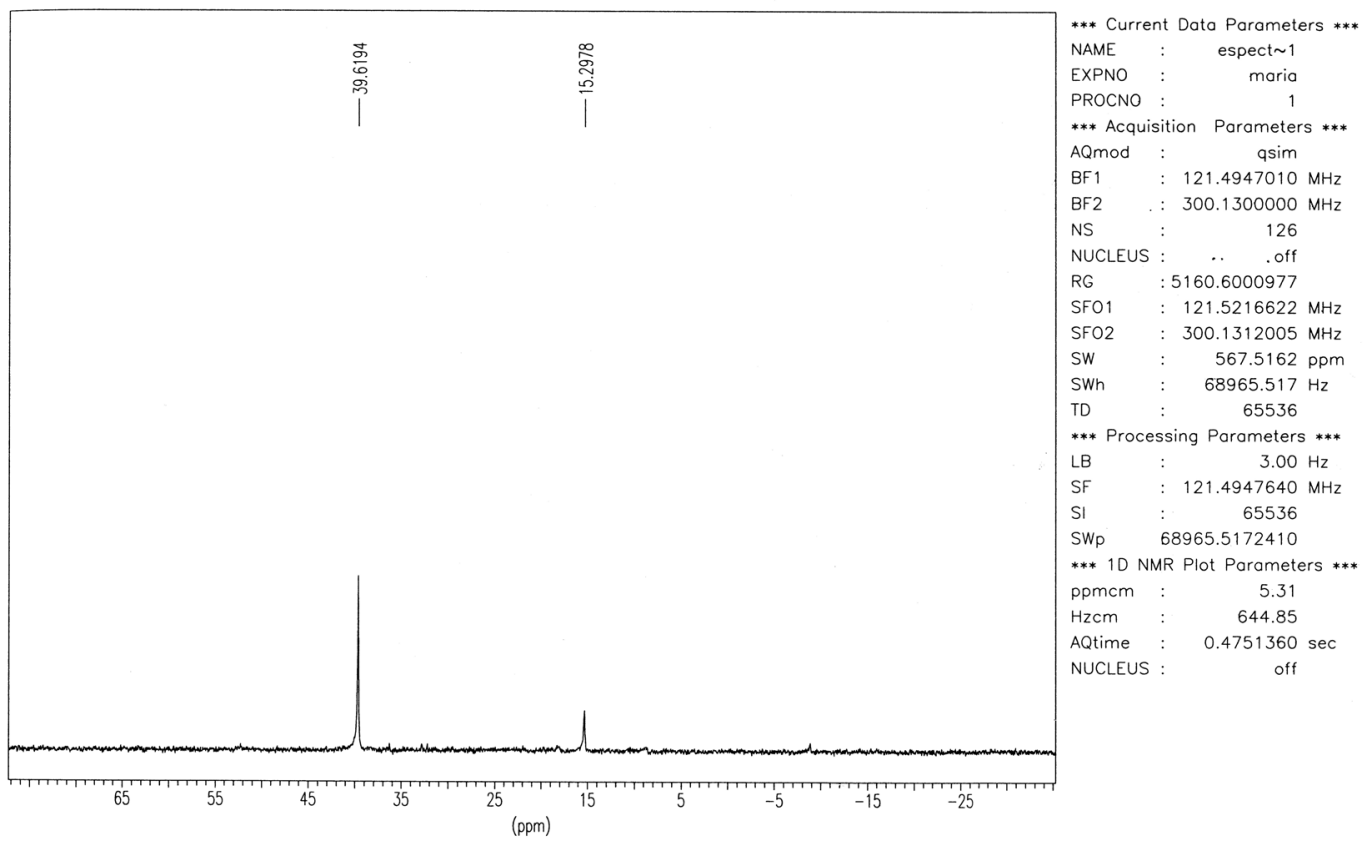

Figure S2. ${ }^{31} \mathrm{P}\left\{{ }^{1} \mathrm{H}\right\}$ NMR spectra of compound $\mathbf{5}$, recorded in $\mathrm{CD}_{2} \mathrm{Cl}_{2}$ solution.(The resonance at $15.3 \mathrm{ppm}$ is due to partial decomposition of $\mathbf{5}$ upon manipulation of its solutions) 